\title{
DEVELOPMENT OF A PULSE VOLTAMMETRIC BIOSENSING DEVICE USING AN ENZYME EMBODIED ELECTRODE
}

\author{
S. Yamauchi, M. Yaoita and Y. Ikariyama*
}

National Rehabilitation Center for the Disabled, Tokorozawa, Saitama 359, Japan * Department of Bioengineering. Tokyo Institute of Technology, Yokohama, Kanagawa 227, Japan

\begin{abstract}
An enzyme embodied electrode (EEE) fabricated by direct immobilization of enzyme on a platinum black electrode was adopted to be used as a working electrode for glucose sensing pulse voltammetric device. A reaction scheme in the micropores of platinum black is proposed to this end. Based on this scheme a preoxidation pulse technique was developed, which was followed by an open circuit period and the measurement with a potentiostatic pulse. A consecutive pulse technique was also proposed, wherein a sequence of an anodic pulse and an open circuit period is repeatedly given. The transient current was measured at each initial $\theta d g \theta$ of the anodic pulse. The open circuit period plays a central role in this pulse technique. A reproducible sensor response with this technique revealed another advantage that any blank measurement are necessary.

Key words biosensor, glucose, pulse voltammetry, enzyme immobilization
\end{abstract}

An enzyme embodied electrode (EEE) is a newly developed amperometric biosensing device based on the direct immobilization of enzyme on an electrode with large specific surface area, especially platinum black[1]. It has be日n developed as a result of our finding that glucose oxidase (GOD) is easily immobilized on the surface of platinum black[2,3]. The direct immobilization ensures a fast response time of less than 3 s[4]. The large surface area of the platinum black gives low detection limit of $0.5 \mu \mathrm{M}$. In addition, it also gives an appreciable current even when a microelectrode down to $1-10 \mu \mathrm{m}$ is employed, and is suitable for construction of microbiosensor. The EEE sensor has also demonstrated a wide dynamic range from $0.5 \mu \mathrm{M}$ to $20 \mu \mathrm{mM}$, and a rapid response time of less than $3 \mathrm{~s}$ in a standard electrochemical cell[4]. The fast response has demonstrated its advantage as a detector of a flow injection analyser, which can measure more than 600 samples an hour[5].

An intriguing application of the EEE sensor is the pulse voltammetric operation[6,7]. We developed a planar electrochemical device, consisting of a glucose sensing EEE as a working electrode, a $\mathrm{Ag} / \mathrm{AgCl}$ reference electrode and a platinum auxiliary electrode in a circle of 3 $\mathrm{mm}$ diameter. One droplet of $2 \sim 20 \mu \mathrm{L}$ sample on this device formed a miniature hydrostatic electrochemical cell. A potentiostatic pulse was applied at $60 \mathrm{~s}$ after the sample was placed, and the resulting transient current was recorded. The difference between the observed current on the glucose solution and that on the blank at 2 ms was taken as a sensor response, which was dependent only on the glucose concentration, irrespective of the sample volume.

Although the above device has demonstrated that a portable glucose sensing device with fast response can be developed, we still have several difficulties. They are:

1) The requirement of a constant waiting time after the sample loading.

2) Subtraction of a transient current of a blank sample.

This paper reports recent progress in an effort toward clinical application. Emphasis is placed on the employment of a combination of a preoxidation pulse, as well as a successive open circuit period to overcome the above difficulties.

\section{EXPERIMENTAL}

Fabrication of the EEE Sensor A platinum wire of $100 \mu \mathrm{m}$ in diameter was sealed in a soda glass capillary tube. The tip of every electrode was polished with alumina powder. After the platinum electrode was first platinized to form platinum black, it was electrochemically passivated at $1.2 \mathrm{~V}$ for $10 \mathrm{~min}$. After the electrode was washed, GOD was immobilized in it by immersion in a phosphate buffered solution containing the enzyme. The microelectrode was then rinsed thoroughly in a $0.1 \mathrm{M}$ phosphate buffer for over 12 hours. The electrode, if necessary, was immersed in a bovine serum albumin solution and then in a glutaraldehyde solution to be covered with a thin protein layer. 
Pulse Voltammetric Measurement The electrode was set in a standard electrochemical cell with a reference electrode of $\mathrm{Ag} / \mathrm{AgCl}$ and a platinum auxiliary electrode. All the measurements were made in a $0.1 \mathrm{M}$ phosphate buffered solution containing $0.05 \mathrm{M} \mathrm{NaCl}$. A potentiostatic pulse of 0.6 V was supplied by a potentiostat for the measurement and preoxidation. The transient current was digitally recorded and the data collected was transferred to a computer.

\section{RESULTS AND DISCUSSION}

Transient Response to a Single Pulse Figure 1 depicts a transient current to a potentiostatic pulse applied at $60 \mathrm{~s}$ after the immersion of the electrode in sample solutions. The response to the blank solution coincided with that to the fructose solution, indicating non-specific response to saccarides exists. The difference of the current for the glucose sample and that for the blank sample can be taken as the sensor response. However, improvement is necessary on this technique to be feasible for practical application, because of the difficulties mentioned earlier. Another disadvantage is that the calibration curve given in Fig. 2 does not liearly depends on the concentration.

Reaction Scheme in a Pulse Voltammetric Operation in order to find out more practically feasible technique we have elucidated a reaction scheme in a platinum black micropore. When an EEE is immersed in an electrochemical cell, glucose diffuses into the micropores. Hydrogen peroxide is produced on an enzymatic oxidation of glucose at the electrode surface. If no potential is applied, it diffuses out of the micropores, as is schematically shown in Fig. 3(A). The concentration profile of hydrogen peroxide in the micropore is established by the above balance. Upon application of an anodic pulse, the hydrogen peroxide is electrochemically oxidized, as illustrated in Fig. 3(B). A transient current represents the oxidation rate of the hydrogen peroxide in the micropores. Therefore, the current indirectly depends on the glucose concentration in the solution.

The above reaction scheme gives an interpretation of the requirement for a constant waiting time after the electrode is in contact with the sample solution. The concentration of the hydrogen peroxide in the micropore depends on both glucose concentration in the solution and the reaction time to produce the hydrogen peroxide. Therefore, one can easily understand that the observed transient current depends on the time after the electrode is immersed in the solution. In other words, reproducible data of the transient current can only be obtained when the reaction time is kept constant.

Single Preoxidation Pulse Technique The above consideration led us to employ a combination of a preoxidation pulse and the successive open circuit period. If we can electrochemically oxidize all the hydrogen peroxide in the micropores, the concentration of hydrogen peroxide will be reduced to be negligibly small. Therefore, we can start the production of hydrogen peroxide at the end of

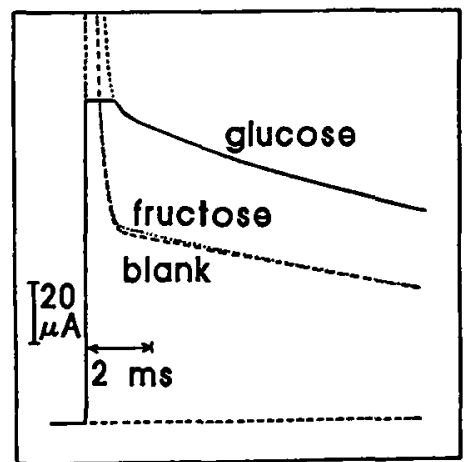

Fig. 1 Transient current to a single pulse

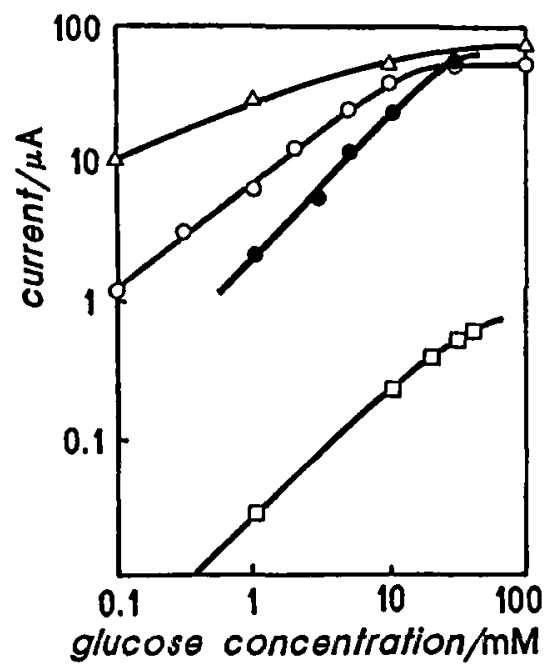

Fig. 2 Calibration curves of the EEE glucose sensor

$O$ : by the single preoxidation pulse

- : by the consecutive pulse

$\triangle$ : by the single pulse

$\square$ : steady current in a standard cell

(A) without a potential

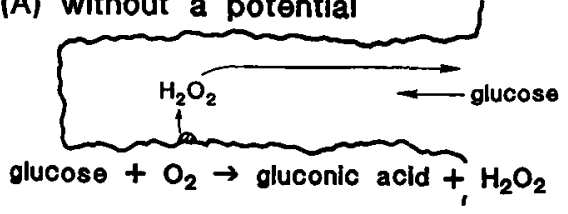

(B) under a constant potential

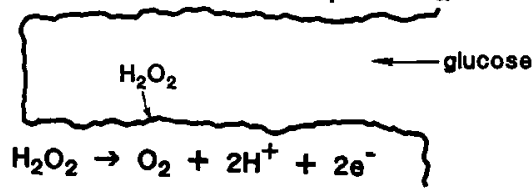

Fig. 3 Production and Decomposition of Hydrogen Peroxide in a micropore 
this oxidation pulse. In order to ensure the

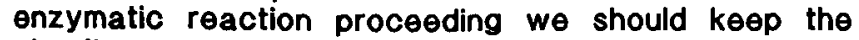
circuit open without an application of any potential. We have further speculated that we can obtain a reproducible transient current by keeping this open circuit period constant after an application of the preoxidation pulse.

In order to confirm the validity of this assumption we made an experiment employing a pulse sequence as illustrated in Fig. 4(A). A preoxidation pulse of $0.6 \mathrm{~V}$ vs $\mathrm{Ag} / \mathrm{AgCl}$ was first applied for a time $t_{\mathrm{p},}$ then the circuit was kept open for a period $t_{0}$. A measurement pulse was finally applied and a transient current was recorded. A typical transient curve is shown in Fig. 5. The current at $10 \mathrm{~ms}$ after the application of the measurement pulse was taken as the sensor response. This response was very reproducible even when no attention was paid to the time after the immersion of the electrode. It is strongly emphasized again that no potential is applied during the open circuit period, because hydrogen peroxide is accumulated in the micropores during this period. This is the central feature of this technique, differing from the standard potential step technique.

The preoxidation technique has offered another improvement. The current for the blank sample was negligibly small when compared to that for the glucose sample as shown by Fig. 5. The current observed for the blank sample in Fig. 1 is an anodic current for the oxidation of a small amount of reducing impurities in the buffered solution and albumin layer. If we kept application of a constant potential in a buffered solution the current would have gradually decreased to a residual current. Normally it takes about an hour before a residual current reaches to the order of several tens of pA. We consider that a preoxidation pulse also oxidizes such impurities and reduces the transient current for a blank sample. From this result we conclude that the single preoxidation pulse technique makes the blank sample measurement unnecessary.

In order to confirm the reaction scheme we examined the effect of the pulse width and the open circuit period to the transient current. Figure 6 shows that the transient current decreased with an increase in the pulse width for preoxidataion. This decrease is explained by the proposed reaction scheme that more hydrogen peroxide is oxidized as the pulse width becomes longer. Whilest the current for the blank sample was negligibly small for a preoxidataion pulse of more than $30 \mathrm{~s}$, the glucose sample gave an appreciable and almost a constant transient current. We consider that the hydrogen peroxide oxidized by the measurement pulse was mainly produced in the open circuit period. If this assumption is valid, the current should increase with an increase in the open circuit period. The results presented in Fig. 7 support this reasoning. We conclude that our reaction scheme has successfully given a qualitative interpretation of the experimental results.

The calibration curve obtained by the single preoxidation pulse technique is compared to that obtained by the previous methods in Fig. 2. The present technique gave a calibration curve almost

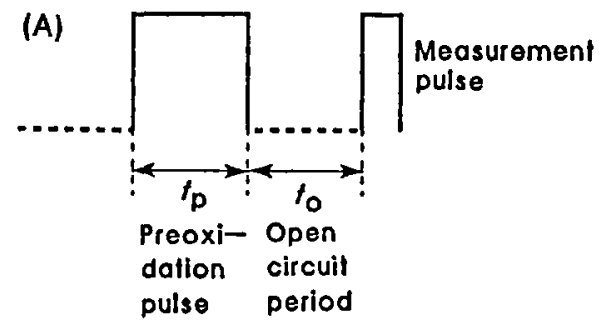

Measurement

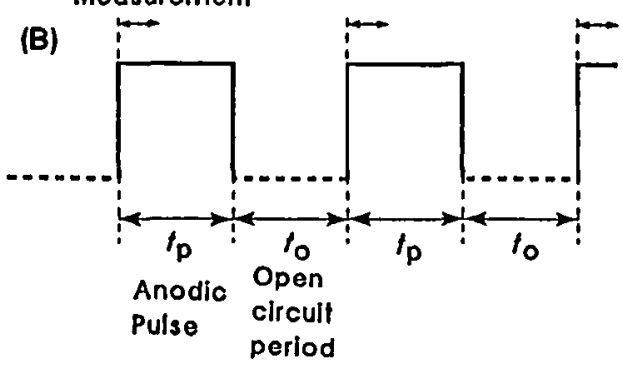

Fig. 4 Pulse sequences for the voltammetric operation

(A) single preoxidation pulse

(B) consecutive pulse

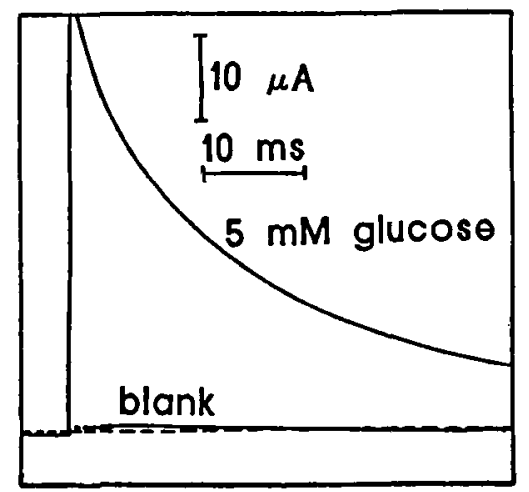

Fig. 5 Typical transient current by a single preoxidation pulse technique ( $\left.t_{p}=60 \mathrm{~s}, t_{0}=10 \mathrm{~s}\right)$

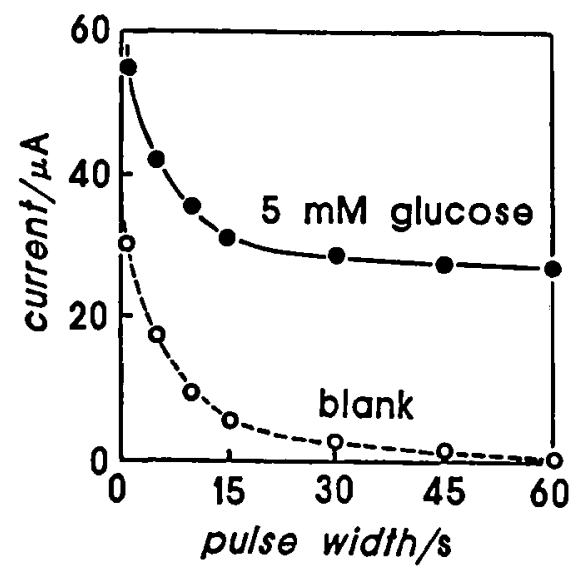

Fig. 6 Dependence of a transient current on the preoxidation pulse width, $t_{\mathrm{p}} \quad\left(t_{0}=10 \mathrm{~s}\right)$ 
one order of magnitude larger than that derived by the measurement of changes in a steady current in a standard cell. Linear dependence on glucose concentration in these results is more suitable for practical clinical use than that measured without the preoxidation technique.

Consecutive Pulse Tochnique Multiple data acquisition and averaging is one of the modern technique employed in a highly accurate measurement. In order to facilitate a signal averaging technique for the pulse voltammetric operation of the EEE sensor, we have adopted a consecutive pulse technique illustrated by Fig. 4(B). In this experiment a potentiostatic anodic pulse of width $t_{\mathrm{p}}$ was repeatedly applied, while the circuit was kept open for a period $t_{0}$ between the pulses. A transient current was measured in the initial period of each anodic pulse and transferred to the computer while the pulse continued. The anodic pulse plays two roles in this experiment. One is to oxidize the hydrogen peroxide and the other is to apply a measurement pulse to obtain the transient current. The current observed for the first pulse is the same with the one for the preoxidation pulse in the single preoxidation pulse technique, and depends on the time after the immersion of the electrode in a sample. It was not reproducible as was expected and was excluded from the signal averaging. In some cases the current to the second pulse was also different from the following ones. Excluding the currents to the first and the second pulses, those to the succeeding pulses were very reproducible between 20 and $30 \mathrm{~ms}$.

The current was not so reproducible during the initial $10 \mathrm{~ms}$, just after the potential was applied. Because the constant potential was applied directly from an open circuit state unlike in a standard potential step method, the potentiostat was not stable in the initial period til about $500 \mu \mathrm{s}$. This instability may have given some influence to the observed current up to $10 \mathrm{~ms}$. It is well known that the rest potential of a platinum electrode in a hydrogen peroxide solution is a mixed potential[8] and is not very stable, especially when the concentration of hydrogen peroxide is low. This electrode process may have also contributed to the scattering in the initial period. Apart from the unstable current in the initial $10 \mathrm{~ms}$, we can expect that a reliable glucose sensing device can be constructed by averaging the reproducible transient current at $20 \sim 30 \mathrm{~ms}$, excluding the current to the initial two pulses.

The calibration curve obtaained by the consecutive pulse technique is also given in Fig. 2. We colclude that linear dependence of the current in the order of $\mu \mathrm{A}$ is feasible for the development of this biosensing device for clinical applications.

\section{REFERENCES}

1. S. Yamauchi, Y. Ikariyama and M. Yaoita, in T. Seiyamaled.), Chemical Sensor Technology, vol. 2, Kodansha Ltd., Tokyo, 1989, pp. 205-223.

2. Y. Ikariyama, S. Yamauchi, T. Yukiashi and H. Ushioda, Anal. Lett., 20, 1407 (1987)

3. Y. Ikariyama, S. Yamauchi, T. Yukiashi and H. Ushioda, Anal. Lett., 20, 1791 (1987)

4. Y. Ikariyama, S. Yamauchi, T. Yukiashi and H. Ushioda, J. Electrochem. Soc., $136,702(1989)$

5. Y. Ikariyama, S. Yamauchi, T. Yuklashi, H. Ushioda and M. Aizawa, Bull. Chem. Soc., Japan, 62, 1869 (1989)

6. Y. Ikariyama, N. Shimada, S. Yamauchi, T. Yukiashi and H. Ushioda, Anal. Lett., $21,953(1988)$

7. Y. Ikariyama, N. Shimada, T. Yukiashi, M. Aizawa and S. Yamauchi, Bull. Chem. Soc., Japan, 62, 1864 (1989)

8. J. P. Hoare, J. Electrochem. Soc., 112, 608 (1965) 